\title{
On the arc filtration for the singularities of Arnold's lists
}

\author{
BY WOLFGANG EBELING \\ Universität Hannover, Institut für Mathematik, \\ Postfach 6009, D-30060 Hannover, Germany. \\ e-mail: ebeling@math.uni-hannover.de \\ AND SABIR M. GUSEIN-ZADE \\ Moscow State University, Faculty of Mechanics and Mathematics, \\ Moscow, 119992, Russia. \\ e-mail: sabir@mccme.ru†
}

(Received 11 September 2003)

\begin{abstract}
In a previous paper, the authors introduced a filtration on the ring $\mathcal{O}_{V, 0}$ of germs of functions on a germ $(V, 0)$ of a complex analytic variety defined by arcs on the singularity and called the arc filtration. The Poincare series of this filtration were computed for simple surface singularities in the 3 -space. Here they are computed for surface singularities from Arnold's lists including uni- and bimodular ones. The classification of the unimodular singularities by these Poincare series turns out to be in accordance with their hierarchy defined by E. Brieskorn using the adjacency relations. We also give a general formula for the Poincaré series of the arc filtration for isolated surface singularities which are stabilizations of plane curve ones.
\end{abstract}

\section{Introduction}

Let $(V, 0)$ be a germ of a complex analytic variety. In [5] we defined a filtration on the ring $\mathcal{O}_{V, 0}$ of germs of functions on the variety $(V, 0)$, which we called arc filtration. An arc $\phi$ on $(V, 0)$ is a germ of a complex analytic mapping $\phi:(\mathbb{C}, 0) \rightarrow(V, 0)$. For an arc $\phi$ on $(V, 0)$ and for a germ $g \in \mathcal{O}_{V, 0}$ let $v_{\phi}(g) \in \mathbb{Z}_{\geqslant 0}$ be the order of the function $g \circ \phi$ at the origin, i.e. the power of the first non-vanishing term in the power series expansion $g \circ \phi(\tau)=a \tau^{v_{\phi}(g)}+\cdots, a \neq 0$ (if $g \circ \phi \equiv 0$, then $v_{\phi}(g)$ is assumed to be equal to $+\infty)$. Let $v(g)$ be the minimum over all arcs $\phi$ on $(V, 0)$ of the orders $v_{\phi}(g)$. The arc filtration $\mathcal{O}_{V, 0}=F_{0} \supset F_{1} \supset F_{2} \supset \cdots$ on the ring $\mathcal{O}_{V, 0}$ is the filtration by the ideals $F_{i}=\left\{g \in \mathcal{O}_{V, 0}: v(g) \geqslant i\right\}$. A filtration defined in a similar way can be constructed on the space of 1 -forms on a singularity.

$\dagger$ Partially supported by the DFG-programme "Global methods in complex geometry" (Eb 102/4-2), grants RFBR-01-01-00739, INTAS-00-259, NSh-1972.2003.1. 
Let $\mathfrak{m}$ be the maximal ideal of the ring $\mathcal{O}_{V, 0}$. Obviously $\mathfrak{m}^{i} \subset F_{i}$ and therefore the ideals $F_{i}$ have finite codimension in the algebra $\mathcal{O}_{V, 0}$. Let

$$
P_{V, 0}(t)=\sum_{i=0}^{\infty} \operatorname{dim}\left(F_{i} / F_{i+1}\right) \cdot t^{i}
$$

be the Poincaré series of the arc filtration on the ring $\mathcal{O}_{V, 0}$.

In [5], the Poincaré series of the arc filtration on the ring of germs of functions of the rational double points were computed. A rational double point is an isolated surface singularity defined by $\{f=0\}$ where $f$ is a simple (0-modular) germ of a function in three variables. For each of these singularities the Poincare series of the arc filtration turns out to coincide with the one of the quasihomogeneous filtration on the ring of functions of another simple surface singularity in $\left(\mathbb{C}^{3}, 0\right)$.

In this paper we continue the study of the Poincare series of the arc filtration of isolated 2-dimensional hypersurface singularities. We compute the Poincare series $P_{V, 0}(t)$ for the following surface singularities of V. I. Arnold's lists [1]: the stabilizations of the curve singularities of multiplicity at most 4 and the singularities of multiplicity 3 with a reduced 3 -jet. These singularities include all the uni- and bimodular surface singularities. For the parabolic singularities we have the following table of Poincaré series $P_{V, 0}(t)$ :

\begin{tabular}{ccc} 
Notation & Equation & $P_{V, 0}(t)$ \\
\hline$T_{2,3,6}\left(J_{10}\right)$ & $x^{6}+y^{3}+z^{2}=0$ & $\frac{1-t^{6}}{(1-t)\left(1-t^{2}\right)\left(1-t^{3}\right)}$ \\
$T_{2,4,4}\left(X_{9}\right)$ & $x^{4}+y^{4}+z^{2}=0$ & $\frac{1-t^{4}}{(1-t)^{2}\left(1-t^{2}\right)}$ \\
$T_{3,3,3}\left(P_{8}\right)$ & $x^{3}+y^{3}+z^{3}=0$ & $\frac{1-t^{3}}{(1-t)^{3}}$ \\
\hline
\end{tabular}

(The notation in brackets is the notation of Arnold [1].) These Poincaré series coincide with the Poincaré series of the natural quasihomogeneous filtrations on the rings of germs of functions on these singularities. We show that the Poincaré series of the arc filtration for any uni- or bimodular surface singularity $(V, 0)$ coincides with one of these series. Moreover, the singularity $(V, 0)$ can be deformed to at least one parabolic singularity [2]. The Poincare series $P_{V, 0}(t)$ of the arc filtration is equal to the corresponding Poincare series of the parabolic singularity with the minimal Milnor number among those to which $(V, 0)$ can be deformed. This means that the classification of the unimodular surface singularities by the Poincare series of their arc filtrations is in accordance with the hierarchy defined by the adjacency relations described by E. Brieskorn [2].

Besides that we give a general formula for the Poincaré series $P_{V, 0}(t)$ for an isolated surface singularity $(V, 0) \subset\left(\mathbb{C}^{3}, 0\right)$ which is the stabilization of a (reduced) plane curve singularity. Moreover, we show that for a hypersurface singularity with a reduced tangent cone the arc filtration coincides with the filtration by powers of the maximal ideal.

\section{Stabilizations of curve singularities}

Since a big part of the singularities from Arnold's lists are stabilizations of plane curve singularities, we start with a study of this case in general. Let $(C, 0) \subset\left(\mathbb{C}^{2}, 0\right)$ be 
a germ of a reduced plane curve given by an equation $f(x, y)=0$ with $f \in \mathcal{O}_{\mathbb{C}^{2}, 0}$. The stabilization of the plane curve singularity $(C, 0)$ is the surface singularity $(S, 0) \subset$ $\left(\mathbb{C}^{3}, 0\right)$ defined by the equation $f(x, y)-z^{2}=0$. The surface $(S, 0)$ has an isolated singularity at the origin. Let $\pi:(\mathcal{X}, \mathcal{D}) \rightarrow\left(\mathbb{C}^{2}, 0\right)$ be an embedded resolution of the curve singularity $(C, 0)$. The exceptional divisor $\mathcal{D}=\pi^{-1}(0)$ of the resolution $\pi$ is the union $\bigcup_{i=1}^{r} E_{i}$ of irreducible components $E_{i}$; each of them is a rational curve, i.e. is isomorphic to the projective line $\mathbb{C P}^{1}$. For a germ $g \in \mathcal{O}_{\mathbb{C}^{2}, 0}, g \neq 0$, let $v_{i}(g)$ be the multiplicity of the lifting $\widetilde{g}=g \circ \pi$ of the function $g$ to the space $\mathcal{X}$ of the resolution $\pi$ along the component $E_{i}$ of the exceptional divisor $\mathcal{D}$. We shall call $m_{i}=v_{i}(f)$ the multiplicity of the component $E_{i}$ of the exceptional divisor ( $f$ is the left-hand side of the equation of the curve $(C, 0)$ ). Let $\sigma_{i}=1$ (respectively $\sigma_{i}=2$ ) if the multiplicity $m_{i}$ is even (respectively odd). Suppose that the resolution $\pi$ has the property that the strict transform $\widetilde{C}$ of the curve $C$ intersects the exceptional divisor $\mathcal{D}$ only at points of the components $E_{i}$ with even multiplicity $m_{i}$. Such a resolution can be obtained from an arbitrary (say, the minimal) one by blowing up intersection points of the strict transform $\widetilde{C}$ with the components $E_{i}$ with odd multiplicity $m_{i}$.

The resolution $\pi$ defines a multi-index filtration on the ring $\mathcal{O}_{\mathbb{C}^{2}, 0}$ of germs of functions of two variables which is called divisorial and which is defined by the valuations $v_{i}(\cdot)$, i.e. the corresponding ideal $J(\underline{v})\left(\underline{v}=\left(v_{1}, \ldots, v_{r}\right) \in \mathbb{Z}^{r}\right)$ is equal to $\left\{g \in \mathcal{O}_{\mathbb{C}^{2}, 0}: v_{i}(g) \geqslant v_{i}, i=1, \ldots, r\right\}$. (Note that we defined the ideal $J(\underline{v})$ for all $\underline{v} \in \mathbb{Z}^{r}$, not only for those with non-negative components.) Let $P_{\pi}\left(t_{1}, \ldots, t_{r}\right)$ be the Poincaré series of this filtration (see, e.g., [4]). The Poincaré series $P_{\pi}\left(t_{1}, \ldots, t_{r}\right)$ is a power series in $t_{1}, \ldots, t_{r}$ defined as

$$
\frac{\left(\sum_{\underline{v} \in \mathbb{Z}^{r}} \operatorname{dim}(J(\underline{v}) / J(\underline{v}+\underline{1})) \underline{\underline{v}}\right) \cdot \prod_{i=1}^{r}\left(t_{i}-1\right)}{t_{1} \cdot \ldots \cdot t_{r}-1} .
$$

Here $\underline{t}^{\underline{v}}=t_{1}^{v_{1}} \cdot \ldots \cdot t_{r}^{v_{r}}, \underline{1}=(1, \ldots, 1) \in \mathbb{Z}^{r}, \sum_{\underline{v} \in \mathbb{Z}^{r}} \operatorname{dim}(J(\underline{v}) / J(\underline{v}+\underline{1})) \underline{t} \underline{\underline{v}}$ is a formal Laurent series in the variables $t_{1}, \ldots, t_{r}$ infinite in all directions for $r \geqslant 2$ (for $r=1$ it is a power series).

For a component $E_{i}$ of the exceptional divisor $\mathcal{D}$ of the resolution $\pi$, let $\stackrel{\circ}{E_{i}}$ be its "smooth part", i.e. $E_{i}$ without the intersection points with other components of the exceptional divisor $\mathcal{D}$, let $\widetilde{L}_{i}$ be a germ of a non-singular curve transversal to the component $E_{i}$ at a smooth point, i.e. at a point of $\stackrel{\circ}{E}_{i}$. Let the blow-down $L_{i}=\pi\left(\widetilde{L}_{i}\right)$ of the curve $\widetilde{L}_{i}$ be given by an equation $g_{i}=0, g_{i} \in \mathcal{O}_{\mathbb{C}^{2}, 0}$, and let $m_{i j}:=v_{j}\left(g_{i}\right)$. The matrix $\left(m_{i j}\right)$ is symmetric and is the inverse to minus the matrix of intersections of the components $E_{i}$. Let $\underline{m}_{i}:=\left(m_{i 1}, \ldots, m_{i r}\right)$. In [4] it was shown that

$$
P_{\pi}\left(t_{1}, \ldots, t_{r}\right)=\prod_{i=1}^{r}\left(1-\underline{t}^{\underline{m_{i}}}\right)^{-\chi\left(\stackrel{\circ}{E}_{i}\right)}
$$

where $\chi(A)$ is the Euler characteristic of a space $A$.

For a power series $P\left(t_{1}, \ldots, t_{r}\right)$, the reduction $\bar{P}(t)$ of this series is the series in one variable $t$ obtained from $P$ by substituting each monomial $\underline{t}^{\underline{k}}, \underline{k}=\left(k_{1}, \ldots, k_{r}\right) \in \mathbb{Z}_{\geqslant 0}^{r}$, by the monomial $t^{k}$ with $k=\min _{1 \leqslant i \leqslant r} k_{i}[5]$. For example, for $P\left(t_{1}, t_{2}\right)=1+t_{1} t_{2}^{2}+$ $t_{1}^{2} t_{2}+t_{1}^{2} t_{2}^{2}$, one has $\bar{P}(t)=1+2 t+t^{2}$. 
Theorem 1. The Poincaré series $P_{S, 0}(t)$ of the arc filtration of the stabilization $(S, 0)$ of the curve $(C, 0)$ is equal to the reduction $\bar{Q}(t)$ of the series

$$
Q\left(t_{1}, \ldots, t_{r}\right)=\left(1+t_{1}^{\sigma_{1} m_{1} / 2} \cdot \ldots \cdot t_{r}^{\sigma_{r} m_{r} / 2}\right) \cdot P_{\pi}\left(t_{1}^{\sigma_{1}}, \ldots, t_{r}^{\sigma_{r}}\right) .
$$

Remark 1. In [5], one finds a somewhat similar formula for the Poincaré series $P_{V, 0}(t)$ for an arbitrary irreducible isolated singularity $(V, 0)$ in terms of its resolution. However, for an arbitrary singularity (in contrast to a plane curve one) sometimes it is difficult to construct a resolution and, moreover, no general formula for the Poincaré series of the corresponding set of divisorial valuations is known.

Remark 2. Though the series $Q\left(t_{1}, \ldots, t_{r}\right)$ depends on $r$ variables, $r$ being the number of irreducible components of the exceptional divisor $\mathcal{D}$, the minimal exponents usually can be met only at variables corresponding to a few first components of the exceptional divisor (in the sense of the order of their appearance in the process of resolution by blow-ups). Often this reduces the calculations considerably.

Example 1. The rational double point $D_{4}$ is the stabilization of the curve singularity $x^{3}+y^{3}=0$. This curve is resolved after blowing up once. However, the exceptional divisor has odd multiplicity (equal to 3 ). Therefore, to get a suitable resolution, one has to blow up the 3 intersection points of the strict transform of the curve with the exceptional divisor. This leads to the resolution with the exceptional divisor consisting of 4 components with the intersection matrix

$$
\left(\begin{array}{cccc}
-4 & 1 & 1 & 1 \\
1 & -1 & 0 & 0 \\
1 & 0 & -1 & 0 \\
1 & 0 & 0 & -1
\end{array}\right)
$$

Therefore

$$
\left(m_{i j}\right)=\left(\begin{array}{llll}
1 & 1 & 1 & 1 \\
1 & 2 & 1 & 1 \\
1 & 1 & 2 & 1 \\
1 & 1 & 1 & 2
\end{array}\right)
$$

and hence

$$
P_{\pi}\left(t_{1}, t_{2}, t_{3}, t_{4}\right)=\frac{1-t_{1} t_{2} t_{3} t_{4}}{\left(1-t_{1} t_{2}^{2} t_{3} t_{4}\right)\left(1-t_{1} t_{2} t_{3}^{2} t_{4}\right)\left(1-t_{1} t_{2} t_{3} t_{4}^{2}\right)} .
$$

Theorem 1 implies that the Poincare series of the arc filtration is the reduction $\bar{Q}(t)$ of the series

$$
\left(1+t_{1}^{3} t_{2}^{2} t_{3}^{2} t_{4}^{2}\right) \frac{1-t_{1}^{2} t_{2} t_{3} t_{4}}{\left(1-t_{1}^{2} t_{2}^{2} t_{3} t_{4}\right)\left(1-t_{1}^{2} t_{2} t_{3}^{2} t_{4}\right)\left(1-t_{1}^{2} t_{2} t_{3} t_{4}^{2}\right)}
$$

which is equal to

$$
\frac{1-t^{3}}{(1-t)^{2}\left(1-t^{2}\right)}
$$

In [5] this result was obtained using a general description of the Poincaré series in several variables for rational surface singularities from [3]. 
The ring $\mathcal{O}_{S, 0}$ of germs of functions on the stabilization $(S, 0)$ is a free $\mathcal{O}_{\mathbb{C}^{2}, 0}$-module of rank 2. Moreover each germ $H \in \mathcal{O}_{S, 0}$ can in a unique way be written in the form $H=h_{1}+z \cdot h_{2}$ with $h_{i} \in \mathcal{O}_{\mathbb{C}^{2}, 0}, i=1,2$. Thus one can identify $\mathcal{O}_{S, 0}$ with $\mathcal{O}_{\mathbb{C}^{2}, 0} \oplus z \cdot \mathcal{O}_{\mathbb{C}^{2}, 0}$. The arc filtration on the ring $\mathcal{O}_{S, 0}$ defines corresponding filtrations on the summands $\mathcal{O}_{\mathbb{C}^{2}, 0}$ and $z \cdot \mathcal{O}_{\mathbb{C}^{2}, 0}$.

Lemma 1. One has $v(H)=\min \left(v\left(h_{1}\right), v\left(z \cdot h_{2}\right)\right)$. Therefore the Poincaré series $P_{S, 0}(t)$ is the sum of the respective Poincaré series of these filtrations on $\mathcal{O}_{\mathbb{C}^{2}, 0}$ and $z \cdot \mathcal{O}_{\mathbb{C}^{2}, 0}$.

Proof. Obviously $v(H) \geqslant \min \left(v\left(h_{1}\right), v\left(z \cdot h_{2}\right)\right)$. Suppose that

$$
v(H)>v:=\min \left(v\left(h_{1}\right), v\left(z \cdot h_{2}\right)\right) .
$$

This implies that there exists an arc $\psi:(\mathbb{C}, 0) \rightarrow(S, 0)$ on $S$ such that $h_{1} \circ \psi(\tau)=$ $a \cdot \tau^{v}+\cdots,\left(z \cdot h_{2}\right) \circ \psi(\tau)=-a \cdot \tau^{v}+\cdots, a \neq 0$. Consider the arc $\psi^{\prime}$ defined by $\psi^{\prime}(\tau)=\iota \circ \psi(\tau)$ where $\iota$ is the natural involution on the surface $S:(x, y, z) \mapsto(x, y,-z)$. Then $v_{\psi^{\prime}}(H)=v$, a contradiction. The statement about the Poincaré series follows from the fact that

$$
\left\{H \in \mathcal{O}_{S, 0}: v(H) \geqslant i\right\}=\left\{h_{1} \in \mathcal{O}_{\mathbb{C}^{2}, 0}: v\left(h_{1}\right) \geqslant i\right\} \oplus\left\{h_{2} \in \mathcal{O}_{\mathbb{C}^{2}, 0}: v\left(z \cdot h_{2}\right) \geqslant i\right\} .
$$

For $h \in \mathcal{O}_{\mathbb{C}^{2}, 0}$, let $\widetilde{v}_{i}(h):=\sigma_{i} \cdot v_{i}(h)$. The functions $\widetilde{v}_{i}(\cdot)$ are not valuations (they do not respect the multiplication), however, they define a multi-index filtration on the space $\mathcal{O}_{\mathbb{C}^{2}, 0}$ as well: $\widetilde{J}(\underline{v})=\left\{h \in \mathcal{O}_{\mathbb{C}^{2}, 0}: \widetilde{v}_{i}(h) \geqslant v_{i}, i=1, \ldots, r\right\}$ for $\underline{v} \in \mathbb{Z}^{r}$.

Let $\widetilde{v}_{i}(z \cdot h):=\sigma_{i} \cdot\left(v_{i}(h)+\left(m_{i} / 2\right)\right)$. Here, with some abuse of notation, one can say that $v_{i}(h)+\left(m_{i} / 2\right)$ is the order along the component $E_{i}$ of the lifting to the space of the resolution of the function $\sqrt{f} \cdot h$. Then the functions $\widetilde{v}_{i}$ define a multi-index filtration on the space $z \cdot \mathcal{O}_{\mathbb{C}^{2}, 0}$ as well.

Lemma 2. One has

$$
v(h)=\min _{1 \leqslant i \leqslant r} \widetilde{v}_{i}(h), \quad v(z \cdot h)=\min _{1 \leqslant i \leqslant r} \widetilde{v}_{i}(z \cdot h) .
$$

Proof. Under the projection $(x, y, z) \mapsto(x, y)$, an arc on the surface $(S, 0)$ is mapped to a plane arc. Moreover, each arc in $\left(\mathbb{C}^{2}, 0\right)$ is the projection of one or two geometrically different arcs on the surface $(S, 0)$. The number of arcs on $(S, 0)$ which are mapped to the same plane arc $\phi:(\mathbb{C}, 0) \rightarrow\left(\mathbb{C}^{2}, 0\right)$ depends on the order $v_{\phi}(f)$ of the function $f$ on the arc $\phi$ and is equal to 1 or 2 if this order is odd or even (and finite) respectively. Moreover, in the first case the are on $(S, 0)$ is a two-fold covering of its projection, in the second case the projection defines an isomorphism between the curve in the plane and each of its preimages. If the order $v_{\phi}(f)$ is infinite, i.e., if the arc $\phi$ lies in the curve $C=\{f=0\}$, the arc $\phi$ has one preimage isomorphic to it.

Let us prove the first statement of the lemma. Let $\widetilde{S} \subset \mathcal{X} \times \mathbb{C}$ be defined by the equation $\widetilde{f}-z^{2}=0$ where $\widetilde{f}=f \circ \pi$ is the lifting of the function $f$ to the space $\mathcal{X}$ of the resolution. The surface $\widetilde{S}$ is a modification of the surface $S$. Arcs on $S$ arriving at the origin are in one-to-one correspondence with arcs on $\widetilde{S}$ arriving at the exceptional divisor $\mathcal{D} \times\{0\}$. If we take an arc on $\mathcal{X}$ which intersects the exceptional divisor $\mathcal{D}$ at a generic point of the component $E_{i}$ then the order of the function $\widetilde{h}$ along a preimage of this arc in $\widetilde{S}$ is just equal to $\widetilde{v}_{i}(h)$. (Note that here we use the fact that the strict transform of the curve $\{f=0\}$ intersects only the components $E_{i}$ of the exceptional divisor $\mathcal{D}$ with even multiplicity $m_{i}$.) Therefore $v(h) \leqslant \min _{1 \leqslant i \leqslant r} \widetilde{v}_{i}(h)$. 
Let us prove the opposite inequality. Suppose that an arc $\phi$ on $\mathcal{X}$ intersects the exceptional divisor $\mathcal{D}$ at a point of $\stackrel{\circ}{E}_{i}$ with multiplicity $\mu_{i} \geqslant 2$. If both $\mu_{i}$ and $m_{i}$ are odd then the order of the function $\widetilde{h}$ along a preimage of the arc $\phi$ in $\widetilde{S}$ is greater than or equal to $2 \mu_{i} v_{i}(h)$ (since the preimage is a two-fold covering of the arc $\phi$ ). If at least one of the numbers $\mu_{i}$ and $m_{i}$ is even then this order is greater than or equal to $\mu_{i} v_{i}(h)$. In any case it is not less than $\widetilde{v}_{i}(h)$.

Suppose that an arc $\phi$ on $\mathcal{X}$ intersects the exceptional divisor $\mathcal{D}$ at the intersection point of two divisors $E_{i}$ and $E_{j}$. After several additional blow-ups of the intersection points of the components of the exceptional divisor, the strict transform of the arc $\phi$ will intersect a new component at a smooth point. Therefore it is sufficient to prove that an additional blow-up of the intersection point of two components (say, $E_{i}$ and $E_{j}$ ) does not change the number $\min _{\{i\}} \widetilde{v}_{i}(h)$. Let the new component created by blowing up be $E_{\alpha}$. Then $v_{\alpha}(h) \geqslant v_{i}(h)+v_{j}(h), m_{\alpha}=m_{i}+m_{j}$. It is not difficult to show that $\widetilde{v}_{\alpha}(h) \geqslant \min \left\{\widetilde{v}_{i}(h), \widetilde{v}_{j}(h)\right\}$ (this can be verified, e.g., considering three cases: (1) both $m_{i}$ and $m_{j}$ are even; (2) both $m_{i}$ and $m_{j}$ are odd; (3) $m_{i}$ is even, $m_{j}$ is odd). This implies the statement.

The proof of the other statement is analogous.

Lemma 3. The Poincaré series of the multi-index filtration on $\mathcal{O}_{\mathbb{C}^{2}, 0}$ (respectively on $\left.z \cdot \mathcal{O}_{\mathbb{C}^{2}, 0}\right)$ defined by the functions $\widetilde{v}_{i}(\cdot)$ is equal to $P_{\pi}\left(t_{1}^{\sigma_{1}}, \ldots, t_{r}^{\sigma_{r}}\right)$ (respectively to $\left.t_{1}^{\sigma_{1} m_{1} / 2} \cdots t_{r}^{\sigma_{r} m_{r} / 2} P_{\pi}\left(t_{1}^{\sigma_{1}}, \ldots, t_{r}^{\sigma_{r}}\right)\right)$.

Proof. A convenient way to see this is to write these Poincaré series as integrals with respect to the Euler characteristic (see, e.g., [4]). For example, the Poincaré series of the multi-index filtration defined by the functions $\widetilde{v}_{i}(\cdot)$ on the space $z \cdot \mathcal{O}_{\mathbb{C}^{2}, 0}$ is equal to

$$
\int_{\mathbb{P O}_{\mathbb{C}^{2}, 0}} t_{1}^{\widetilde{v}_{1}(z h)} \cdots t_{r}^{\widetilde{v}_{r}(z h)} d \chi .
$$

Comparison of these integrals gives the statement.

Proof of Theorem 1. The Poincaré series of the one-index filtration defined by the function $\min _{1 \leqslant i \leqslant r} \widetilde{v}_{i}(\cdot)$ is the reduction of the (multi-variable) Poincare series of the multi-index filtration defined by the functions $\widetilde{v}_{i}(\cdot)$. (This can be derived using the same arguments as in [5, proposition 2].) Now the statement follows from Lemmas 1-3.

As a corollary of Theorem 1 one has the following statement. For a germ $f \in \mathcal{O}_{\mathbb{C}^{2}, 0}$ let $m(f)$ be its multiplicity, i.e. $f \in \mathfrak{m}^{m(f)} \backslash \mathfrak{m}^{m(f)+1}$. Suppose that an irreducible curve is tangent to a smooth one. In local coordinates $(u, v)$ with the $u$-axis representing the smooth curve the first one can be given by an equation $v=p(u)$ where $p(u)$ is a Puiseux series. We call the first non-trivial exponent of this series the order of contact between the curves.

Corollary 1. (i) If $m(f)$ is even then

$$
P_{S, 0}(t)=\frac{1-t^{m}}{\left(1-t^{m / 2}\right)(1-t)^{2}} .
$$

(ii) Let $m(f)$ be odd and let all components of the curve $C$ be tangent to the same smooth 
curve with order of contact at least 2 . Then

$$
P_{S, 0}(t)=\frac{1-t^{2 m}}{\left(1-t^{m}\right)\left(1-t^{2}\right)(1-t)} .
$$

Proof. Let us number the components of the exceptional divisor in the order of their appearance in the process of the resolution by blow-ups. If $m(f)$ is even the statement follows from the fact that $m_{1}=m(f), \sigma_{1}=1$, and for any $i, j$ one has $m_{i 1}=1 \leqslant m_{i j}$. If $m(f)$ is odd then $m_{1}=m(f), m_{2}=2 m(f), m_{i 1}=1, m_{i 2}=2$ for $i \geqslant 2$ and $m_{i j} \geqslant m_{i 2}$ for $j \geqslant 2$. This implies the statement.

\section{Singularities with a reduced tangent cone}

Let a hypersurface singularity $(V, 0) \subset\left(\mathbb{C}^{n+1}, 0\right)$ be given by an equation $f=0$ with $f=f_{d}+f_{d+1}+\cdots$ where $f_{d}$ is the homogeneous part of $f$ of degree $d, f_{d} \neq 0$. The tangent cone to the singularity $(V, 0)$ is given by the equation $f_{d}=0$.

Theorem 2. If the tangent cone is reduced then the arc filtration on the ring $\mathcal{O}_{V, 0}$ coincides with the filtration by powers of the maximal ideal. Therefore

$$
P_{V, 0}(t)=\frac{1-t^{d}}{(1-t)^{n+1}} .
$$

Proof. Obviously $\mathfrak{m}^{i} \subset F_{i}$. To prove that $\mathfrak{m}^{i}=F_{i}$ we shall show that for $h \in \mathfrak{m}^{i} \backslash \mathfrak{m}^{i+1}$ we have $v(h)=i$. This follows from the fact that there are many smooth arcs on the hypersurface $V$. The fact that $h \in \mathfrak{m}^{i} \backslash \mathfrak{m}^{i+1}$ means that a representative of $h$ in $\mathcal{O}_{\mathbb{C}^{n+1}, 0}$ (which we also denote by $h$ ) can be chosen of the form $h=h_{i}+h_{i+1}+\cdots$ where the homogeneous part $h_{i}$ of degree $i$ is not divisible by $f_{d}$. Therefore there is a regular point $p$ in the hypersurface $\left\{f_{d}=0\right\} \subset \mathbb{C P}^{n}$ such that $h_{i}(p) \neq 0$. After blowing up the origin in $\mathbb{C}^{n+1}$, the strict transform $\widetilde{V}$ of the hypersurface $V$ is non-singular at the point $p$ of the exceptional divisor $\mathbb{C P}^{n}$ and intersects $\mathbb{C P} \mathbb{P}^{n}$ at this point transversally. An arc in the strict transform $\widetilde{V}$ transversal to $\mathbb{C P}^{n}$ blows down to a smooth arc along which the order of the function $h$ is equal to $i$. Therefore $v(h) \leqslant i$.

Example 2. In particular, Theorem 2 implies the following statement: if a hypersurface singularity $(V, 0)$ is the double suspension of a hypersurface singularity $\{f=0\} \subset \mathbb{C}^{n}$ with $f \in \mathfrak{m}^{2}$ (i.e. it is the hypersurface singularity in $\mathbb{C}^{n+2}$ defined by the equation $\left.f\left(z_{1}, \ldots, z_{n}\right)+x^{2}+y^{2}=0\right)$ then

$$
P_{V, 0}(t)=\frac{1-t^{2}}{(1-t)^{n+2}}
$$

$[5$, proposition 2].

Example 3. If $f(x, y, z)=x y z+$ terms of higher degree then, for the surface singularity $V=\{f=0\}$, one has

$$
P_{V, 0}(t)=\frac{1-t^{3}}{(1-t)^{3}} .
$$




\section{Singularities of Arnold's lists}

Theorem 3. The Poincaré series $P_{V, 0}(t)$ of the arc filtration are given by the following list.

(i) For the stabilizations of the curve singularities with non-zero 3-jet except the simple ones, i.e. $J_{k, i}(i \geqslant 0), E_{6 k}, E_{6 k+1}$ and $E_{6 k+2}($ in all cases $k \geqslant 2)$, we have

$$
P_{V, 0}(t)=\frac{1-t^{6}}{(1-t)\left(1-t^{2}\right)\left(1-t^{3}\right)} \text {. }
$$

(ii) For the stabilizations of the curve singularities with zero 3-jet and non-zero 4-jet, i.e. for those of classes $\mathbf{X}, \mathbf{Y}, \mathbf{Z}$ and $\mathbf{W}$, we have

$$
P_{V, 0}(t)=\frac{1-t^{4}}{(1-t)^{2}\left(1-t^{2}\right)}
$$

(iii) For the singularities of multiplicity 3 with a reduced 3 -jet, i.e. for those of the series $\mathbf{Q}, \mathbf{S}$ and $\mathbf{U}$, we have

$$
P_{V, 0}(t)=\frac{1-t^{3}}{(1-t)^{3}} .
$$

Proof. Statement (i) is a particular case of Corollary 1 with $m=3$. Statement (ii) is a particular case of this corollary with $m=4$. Finally, (iii) is a particular case of Theorem 2.

\section{REFERENCES}

[1] V. I. Arnold, S. M. Gusein-Zade and A. N. Varchenko. Singularities of Differentiable Maps, Vol. I (Birkhäuser, 1985).

[2] E. Brieskonn. Die Hierarchie der 1-modularen Singularitäten. Manuscripta Math. 27 (1979), $183-219$.

[3] A. Campillo, F. Delgado and S. M. Gusein-Zade. Poincaré series of a rational surface singularity. Inventiones Math. 155 (2004), 41-53.

[4] F. Delgado and S. M. Gusein-Zade. Poincaré series for several plane divisorial valuations. Proc. Edinburgh Math. Soc. 46 (2003), 501-509.

[5] W. Ebeling and S. M. Gusein-Zade. A filtration defined by ares on a variety. Preprint 2003, math.AG/0303331. 